OPEN ACCESS

Edited by: Kevin Lu,

University of South Carolina,

United States

Reviewed by:

Robert Drury,

Canary Systems, United States

Hong Zhang,

Shanghai University of Traditional

Chinese Medicine, China

${ }^{*}$ Correspondence: Fu Peng

fujing126@yeah.net Gang Wang

wgang@scu.edu.cn

Chenghao Yu

yuholy@126.com

Specialty section:

This article was submitted to

Cancer Molecular Targets and Therapeutics,

a section of the journa

Frontiers in Oncology

Received: 21 June 2021 Accepted: 08 October 2021

Published: 26 October 2021

Citation:

Feng Y, Deng L, Guo H, Zhao Y, Peng F, Wang $G$ and $Y u C$ (2021) The Anti-Colon Cancer Effects of Essential Oil of Curcuma phaeocaulis Through

Tumour Vessel Normalisation.

Front. Oncol. 11:728464. doi: 10.3389/fonc.2021.728464

\section{The Anti-Colon Cancer Effects of Essential Oil of Curcuma phaeocaulis Through Tumour Vessel Normalisation}

\author{
Yewen Feng ${ }^{1}$, Lu Deng ${ }^{2}$, Hengrui Guo ${ }^{3}$, Yumin Zhao ${ }^{1}$, Fu Peng ${ }^{4 *}$, Gang Wang ${ }^{2 *}$ \\ and Chenghao $\mathrm{Yu}^{1 *}$
}

1 State Key Laboratory of Southwestern Chinese Medicine Resources, School of Basic Medicine, Chengdu University of Traditional Chinese Medicine, Chengdu, China, ${ }^{2}$ National Engineering Research Center for Biomaterials, Sichuan University, Chengdu, China, ${ }^{3}$ Southwest Jiaotong University, Chengdu, China, ${ }^{4}$ West China School of Pharmacy, Sichuan University, Chengdu, China

Background: Normalising tumour vessels had become a significant research focus in tumour treatment research in recent years. Curcumae rhizoma (CR) is an essential plant in traditional Chinese medicine as it promotes blood circulation and removes blood stasis. Similarly, CR improves local blood circulation.

Purpose: We explored the anti-colon cancer effects of essential oil from CR (OCR) by investigating its role in normalising tumour vessels. We also provided a basis for research and development into new anti-cancer drugs.

Methods: We used colon cancer as a research focus to investigate OCR. We established an in vitro co-culture model of colon cancer cells and human umbilical vein endothelial cells (HUVEC). We also established an in vivo subcutaneous implant colon cancer model in nude mice. These studies allowed us to evaluate the comprehensive effects of OCR in in vivo and in vitro colon cancer and its role in normalising tumour blood vessels.

Results: In vitro, we found that OCR inhibited Human colon cancer cells (HCT116) and HUVEC cell proliferation and inhibited vascular endothelial growth factor-a (VEGFa) mRNA and protein expression in HUVECs in a co-culture system. Our in vivo studies showed that OCR inhibited colon cancer tumour growth, reduced angiogenesis in tumours and increased vascular endothelial (VE)-cadherin and pericyte coverage in tumour vessels.

Conclusions: OCR inhibited colon cancer growth both in in vivo and in vitro models, reduced angiogenesis in tumours, improved tumour vessel structures and normalised tumour vessels.

Keywords: Curcumae rhizoma, tumour vessel, normalisation, colon cancer, vessel structure

Abbreviations: CR, Curcumae Rhizoma; OCR, essential oil from CR; HUVEC, Human umbilical vein endothelial cells; VEGFa, Vascular endothelial growth factor-a. 


\section{BACKGROUND}

Angiogenesis is an important physiological process during embryonic development, growth and tissue repair. Imbalanced angiogenesis can lead to various pathological consequences and is a particular hallmark feature of tumours (1). From an anatomical perspective, tumour blood vessels are generally disorderly; their generation and distribution are not controlled by normal physiology, leading to spatial abnormalities in blood flow. At the cellular level, the vascular endothelium and pericytes in tumours are missing and cause serious leakage of vascular contents (2-4). This alteration exacerbates local hypoxia and acidosis in tumour environments, eventually converting tumour cells to malignant phenotypes and affecting the killing capacity of immune cells and therapeutics toward tumours (5).

For these reasons, studies have focused on tumour vessel biology. In 1971, researchers pioneered starving tumours to death (6), where tumour formation was inhibited by inhibiting tumour angiogenesis. However, over-pruning tumour vessels had the same effect as blood vessels' overgrowth. Tumour vessels cannot achieve their normal vascular functions (7). After this, scientists turned their attention to normalising tumour vessels. By regulating the balance of pro- and anti-angiogenic factors, tumour vessel functions and structures could be normalised (8). Recent studies showed that particular drugs could reduce tumour metastases, enhance chemotherapeutic drug delivery and increase the anti-cancer effects of chemotherapeutic drugs after effective vascular normalisation (9-12).

In recent years, bioactive compounds from traditional Chinese medicine and associated derivatives have gained scientific acceptance as potentially promising complementary and alternative medicines to treat various diseases (13-16).

Curcumae rhizoma (CR) is the dried rhizome of Curcuma phaeocaulis Valeton, Curcuma kwangsiensis S. G. Lee et C. F. Liang or Curcuma wenyujin Y. H. Chen et C. Ling plant (13). Traditional Chinese medicine proposes that local tumours suffer from stagnation of 'qi', blood and poor metabolism (17). Therefore, for tumour treatment, drugs that promote blood circulation and remove blood stasis are combined to improve local blood circulation. CR is an important traditional Chinese plant that promotes blood circulation and removes blood stasis.

The essential oil from CR (OCR) is the main medicinal active ingredient. The main components are ketones, olefins and aromatic hydrocarbons. Of these, furanodiene, furanodienone, curcumol, $\beta$-elemene, curedione and gemmazone are more abundant $(17,18)$. Studies have shown that CR reduces blood viscosity and improves blood flow rates (19). In addition, evidence suggests that $\beta$-elemene increases (VE)-cadherin expression in tumour tissues (20). For these reasons, it is hypothesised that OCR normalises blood vessels in colon cancer tumours.

In this study, we examined OCR's effects on cell viability, proliferation and vascular endothelial growth factor-a (VEGFa) expression in cultured cell lines human colon cancer cells (HCT116) and human umbilical vein endothelial cells
(HUVEC)) in vitro. In addition, we simulated HUVEC growth in a tumour environment in vitro to study the effects of OCR on the expression of key angiogenic factors. Then, the inhibitory effects of OCR on colon cancer and its effects on tumour vessels were studied in vivo. We aimed to explore the promotion of blood circulation to remove blood stasis and provide a basis for developing potential new tumour drugs for colon cancer.

\section{METHODS}

\section{Materials}

Cells: HUVECs and HCT116 cells were obtained from the National Engineering Research Centre for Biomaterials of Sichuan University.

Animals: Four-week-old male BALB/C-NU mice were purchased from Sibefu Biotechnology Co., Ltd. (Experimental Animal Licence: SCXK (Beijing) 2019-0010). Nude mice were adaptively fed for one week. Studies were conducted per protocols approved by the Animal Ethics Committee of Chengdu University of Traditional Chinese Medicine (Animal Ethics Approval Number: 2017-08).

Drugs: OCR was diluted in normal saline (Chroma, Chengdu, China). The daily dose was $0.1 \mathrm{mg} / \mathrm{g}$. Oxaliplatin (Solarbio, Beijing, China) was diluted in a $5 \%$ glucose solution.

\section{Cell Culture}

HCT116 cells and HUVECs were cultured in RPMI-1640 (Gibco, USA) complete medium containing $10 \%$ foetal bovine serum (Gibco) at $37^{\circ} \mathrm{C}$ in a $5 \% \mathrm{CO}_{2}$ environment. The indirect co-cultivation system method is adopted to simulate the tumor environment. HCT116 and HUVEC were seeded on the upper or lower layers of the transwell chamber, and the two kinds of cells were placed in the same culture environment. After $24 \mathrm{~h}$ of different drug intervention, characterisation studies were performed.

\section{Animal Studies}

In vivo studies: Animals were adaptively fed for one week, after which a colon cancer subcutaneous transplantation model was prepared. HCT116 cells were prepared at $1.5 \times 10^{6}$ cells $/ 100 \mu \mathrm{l}$ normal saline. A $1 \mathrm{ml}$ injection needle was used to subcutaneously inject $100 \mu \mathrm{l}$ cell suspension into the armpit of mice. Tumour size was measured using a Vernier calliper every three days and tumour volumes calculated according to the formula; $\mathrm{V}=1 / 2 \times$ length $\times$ width $^{2}$.

When the average tumour volume was $>100 \mathrm{~mm}^{3}$, we randomly divided the mice into four groups; OCR, OCR + oxaliplatin, oxaliplatin only and model groups. Mice in OCR and OCR + oxaliplatin groups received daily OCR intragastric doses (Figure 1). Mice in OCR + oxaliplatin and oxaliplatin groups received intraperitoneal injections with oxaliplatin every 3 days. The model group received no treatment. After study completion, the mice were anesthetised with $2 \%$ sodium pentobarbital $(0.25$ $\mathrm{ml} / 100 \mathrm{~g}$ ) and sacrificed by cervical dislocation, and subcutaneous tumours removed for immunofluorescence. 


\section{HCT116 implantation to 5-week-old BALB/C-NU male mice
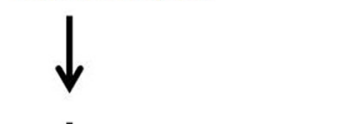

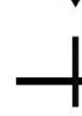

DAY 0

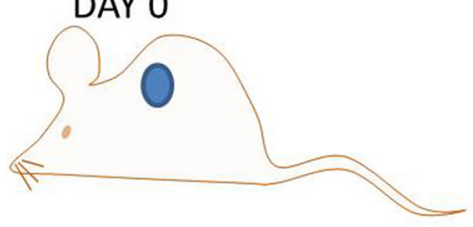

OCR Group: OCR $(0.1 \mathrm{mg} / \mathrm{kg} /$ day $)$

OCR+Oxaliplatin Group:

OCR $(0.1 \mathrm{mg} / \mathrm{kg})+$ Oxaliplatin $(3 \mathrm{mg} / \mathrm{kg})$

Oxaliplatin Group: Oxaliplatin $(3 \mathrm{mg} / \mathrm{kg})$

Control Group: No treatment

FIGURE 1 | Animal study design.

\section{CCK-8 Assay}

Cells were seeded in 96-well plates at appropriate densities. After drug intervention, a complete medium containing 10\% CCK- 8 (Meilunbio, Dalian, China) was added to well for $1 \mathrm{~h}$ in the dark. Optical density values at $450 \mathrm{~nm}$ were measured using a microplate reader. Cell viability was then calculated.

\section{The 5-Ethynyl-2'-Deoxyuridine Assay}

Cells were seeded in 6-well plates at appropriate densities. After a $24 \mathrm{~h}$ drug treatment, an EdU kit (Meilunbio) was used for staining. Cells were observed under a microscope (green fluorescencel blue fluorescence).

\section{Quantitative Polymerase Chain Reaction}

After extracting RNA, reverse transcription was performed using the HiScript III RT SuperMix kit (Vazyme, Nanjing, China). Then enzyme (Vazyme) was added and PCR instrumentation used (QuantStudio 3, Thermo) for amplification.

\section{Western Blotting}

After lysing cells, lysates were centrifuged and supernatants assayed for protein determination using the bicinchoninic acid (BCA) protein quantification kit (Thermo Fisher Scientific). VEGFa expression (Abcam) was detected, and $\beta$-actin (Proteintech, Wuhan, China) used as a loading control.

\section{Immunofluorescence}

Fresh tumour tissue was embedded in paraffin and sectioned. Sections were subjected to antigen retrieval and incubated with CD31 (Servicebio, Wuhan, China), anti- platelet-derived growth factor receptor- $\beta$ (PDGFR- $\beta$ ) (Abcam) and CD144 antibodies (Thermo) which was used to label vascular endothelial (VE)cadherin depending on the investigation. Images were viewed under a microscope and acquired and processed using.

\section{Statistical Analysis}

Data were processed using a one-way analysis of variance. A $\mathrm{P}$ value $<0.05$ was considered statistically significant. Data were displayed as the mean \pm standard deviation (SD).

\section{RESULTS}

\section{The Effects of OCR on HCT116 Cells}

Different OCR concentrations were incubated with HCT116 cells for a $24 \mathrm{~h}$ period and cell viability assessed using the CCK-8 method. HCT116 viability decreased with increasing OCR concentrations. HCT116 cells were less tolerant to OCR at $\mathrm{IC}_{50}=678.5 \mu \mathrm{g} / \mathrm{ml}$ (Figures 2A, B). Based on the OCR intervention curve, two safe doses were selected for drug intervention follow-up studies; a low concentration dose of 20 $\mu \mathrm{g} / \mathrm{ml}$ and a medium concentration dose of $50 \mu \mathrm{g} / \mathrm{ml}$.

To observe the effects of OCR on HCT116 proliferation, we pre-treated the cells with low $(20 \mu \mathrm{g} / \mathrm{ml}$ : HCT116 20 group $)$ and medium OCR concentrations ( $50 \mu \mathrm{g} / \mathrm{ml}$ : HCT116 50 group) for $24 \mathrm{~h}$, and the EdU assay performed to assess proliferation. EdU staining showed that the ratio of EdU-labelled cells at $24 \mathrm{~h}$ for both experimental groups was significantly lower than the HT116 control group $(\mathrm{P}<0.001)$. In addition, the ratio of EdU-labelled cells in the HCT116 50 group at $24 \mathrm{~h}$ was significantly lower than the HCT116 20 group $(\mathrm{P}<0.05)$ (Figures 2C, D). These data suggested that the inhibitory effects of OCR in HCT116 cells were concentration-dependent.

To observe the effects of OCR on VEGFa mRNA expression in HCT116 cells, we pre-treated the cells with low and medium OCR concentrations for $24 \mathrm{~h}$, after which VEGFa mRNA expression was quantified. We observed no significant differences in expression between groups, including the control group (Figure 2E). 


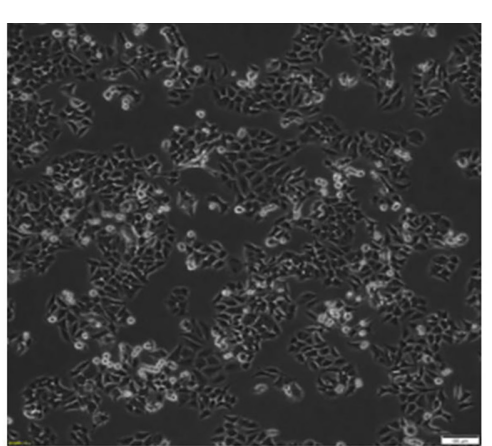

C
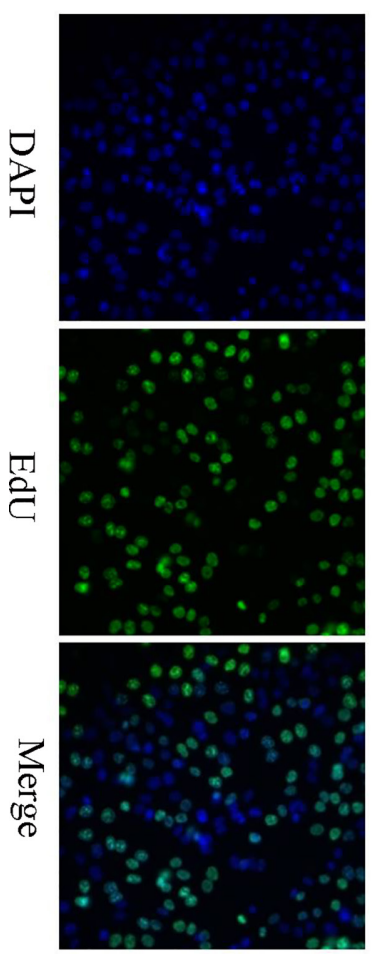

HCT116 Control
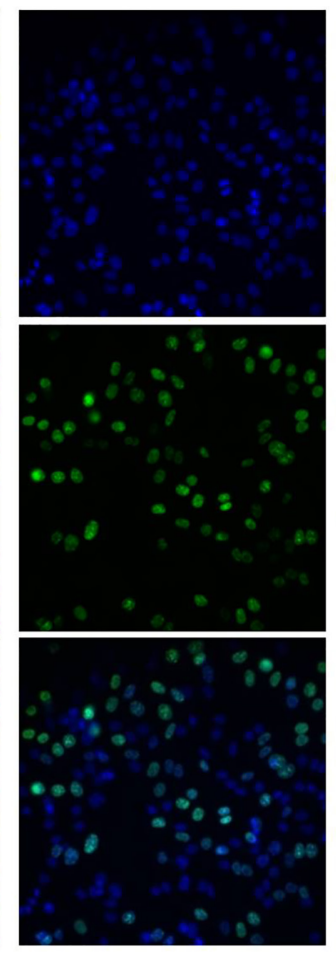

HCT116 20

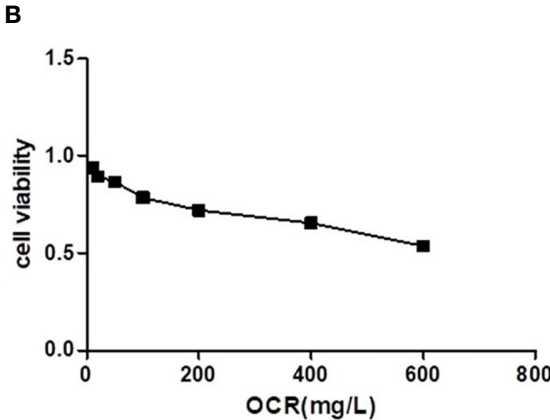

D
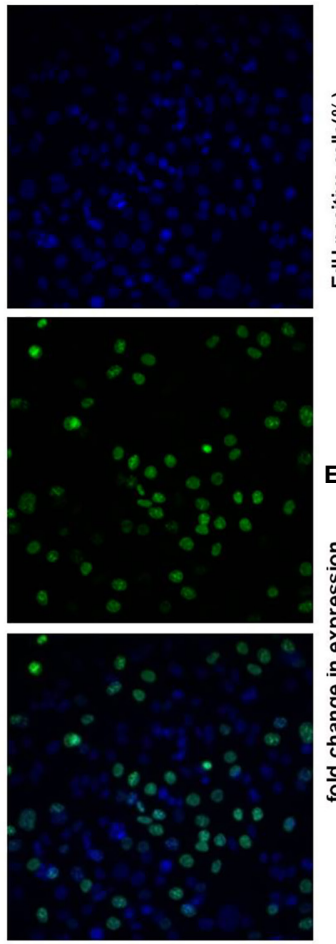

HCT116 50

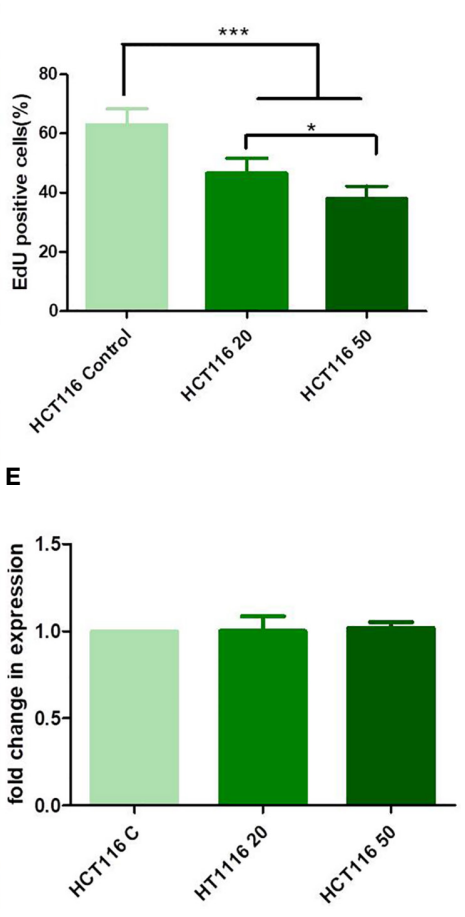

FIGURE 2 The effects of essential oil from Curcumae rhizoma (CR) (OCR) on HCT116 cells. (A) HCT116 cell morphology. (B) The effects of different OCR concentrations on HCT116 viability. (C, D) The effects of OCR on HCT116 proliferation. (E) The effects of OCR on VEGFa mRNA expression in HCT116 cells. ( $\mathrm{n}=3$, mean \pm standard deviation (SD), ${ }^{* *} \mathrm{P}<0.001,{ }^{*} \mathrm{P}<0.05$ compared with the control group).

\section{The Effects of OCR in HUVECs}

Different OCR concentrations were incubated with HUVECs for a 24 $\mathrm{h}$ period and cell viability assessed using the CCK- 8 method. HUVEC viability decreased with increasing OCR concentration. When compared with HCT116 cells, HUVECs were more resistant to OCR, with an $\mathrm{IC}_{50}=950 \mu \mathrm{g} / \mathrm{ml}$ (Figures 3A, B). Based on the OCR intervention curve, two relatively safe doses were selected for drug intervention follow-up studies with HUVECs; a low concentration dose of $50 \mu \mathrm{g} / \mathrm{ml}$ and a medium concentration of $150 \mu \mathrm{g} / \mathrm{ml}$.

To observe the effects of OCR on HUVEC proliferation, we pre-treated the cells with low $(50 \mu \mathrm{g} / \mathrm{ml}$ : OCR 50 group) and medium OCR concentrations ( $150 \mu \mathrm{g} / \mathrm{ml}$ : OCR 150 group) for
$24 \mathrm{~h}$. EdU staining showed that the ratio of EdU-labelled cells at $24 \mathrm{~h}$ in the OCR 50 group was not significantly different to the control group. However, the ratio of EdU cells in the OCR 150 group was significantly lower than both control $(\mathrm{P}<0.001)$ and OCR50 groups $(\mathrm{P}<0.001)$ (Figures 3C, D).

To determine the effects of OCR on VEGFa RNA expression in HUVECs, we pre-treated the cells with low and medium OCR concentrations for $24 \mathrm{~h}$, after which expression was quantified. VEGFa mRNA expression in both experimental groups were significantly lower than the control group $(\mathrm{P}<0.05)$ (Figure 3E).

To observe the effects of OCR on VEGFa protein expression, HUVECs were pre-treated with low and medium OCR 
A

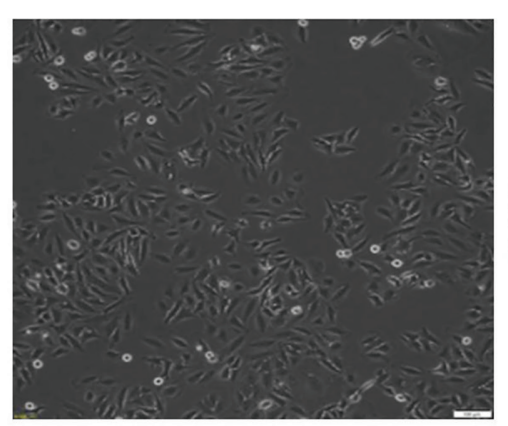

B

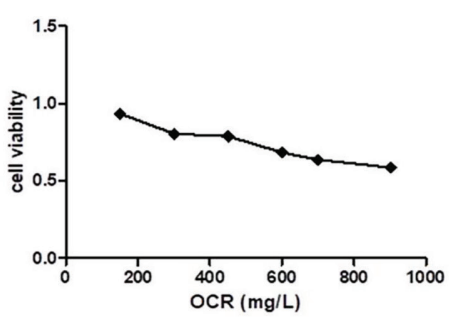

C
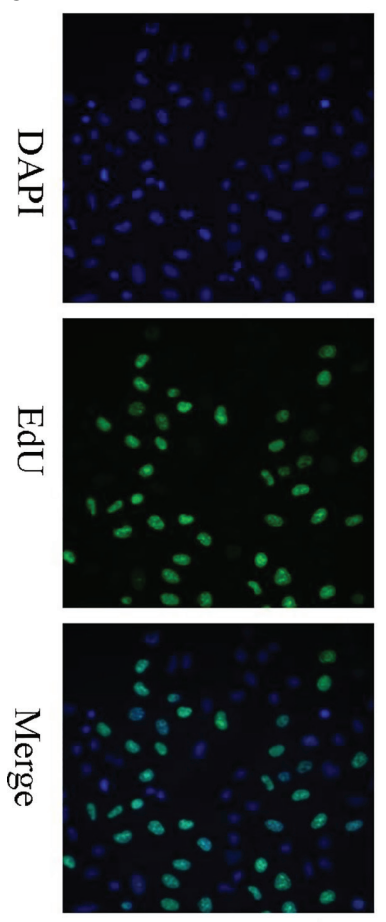

OCR Control
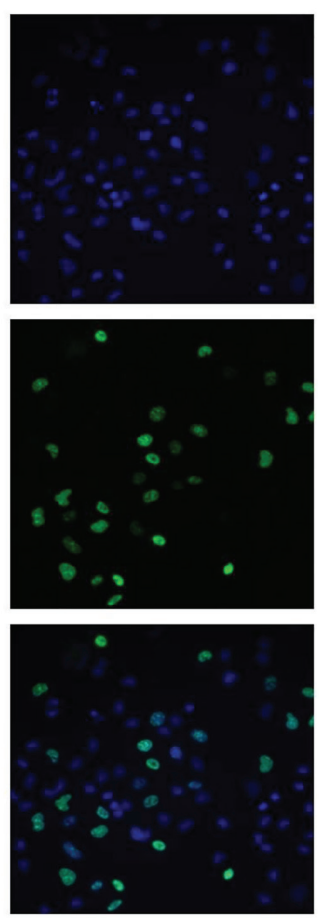

OCR 50
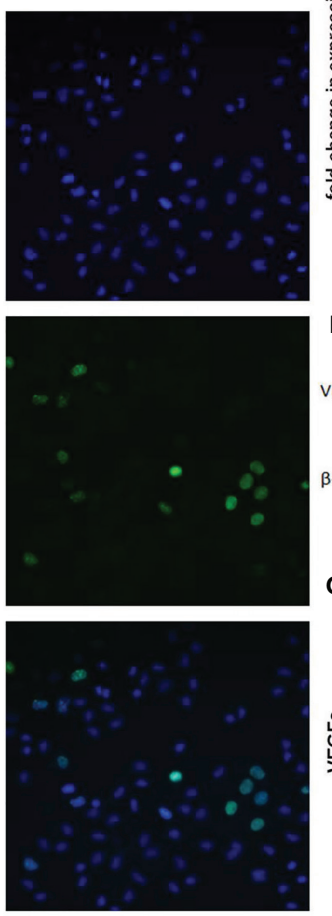

OCR 150

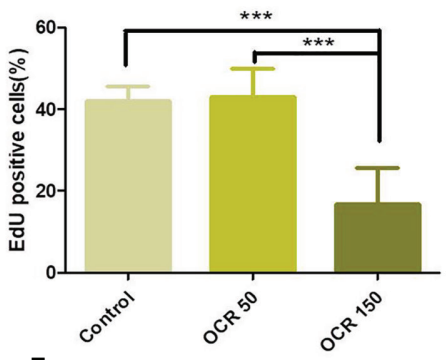

E
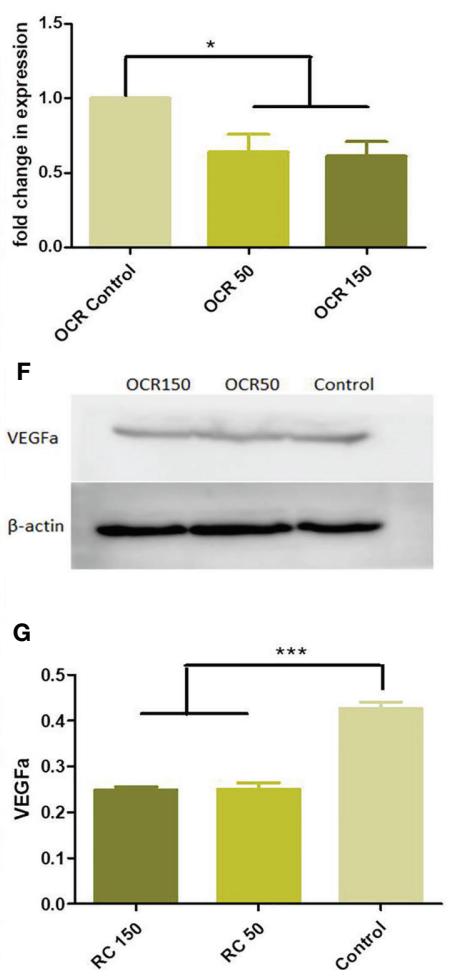

FIGURE 3 | The effects of essential oil from Curcumae rhizoma (CR) (OCR) on HUVECs. (A) HUVEC morphology. (B) The effects of different OCR concentrations on HUVEC viability. (C, D) The effects of OCR on HUVEC proliferation. (E) The effects of OCR on VEGFa mRNA expression in HUVECs. (F, G) The effects of OCR on VEGFa protein expression in HUVECs. ( $n=3$, mean \pm standard deviation (SD), ${ }^{\star * *} \mathrm{P}<0.001,{ }^{*} \mathrm{P}<0.05$ compared with the control group).

concentrations for $24 \mathrm{~h}$, after which western blotting was performed. VEGFa protein expression in both experimental groups was significantly lower than the control group $(\mathrm{P}<0.05)$ (Figures 3F, G).

\section{The Effects of OCR on HUVECs Co- Cultured With HCT116 Cells}

To observe the effects of OCR on HUVEC morphology in a coculture system, we incubated different OCR concentrations (20 $\mu \mathrm{g} / \mathrm{ml}, 50 \mu \mathrm{g} / \mathrm{ml}$ and $150 \mu \mathrm{g} / \mathrm{ml}$ ) with HUVECs in a co-culture system for $24 \mathrm{~h}$, after which cell morphology was investigated. HUVECs in the co-cultured control group exhibited an irregular cell morphology, longer cell antennae and poor general cell status. In contrast, HUVEC morphology in the co-culture system plus OCR supplementation was relatively regular and cells were in good general condition (Figure 4A).

To quantify the effects of OCR on VEGFa mRNA expression in HUVECs in the co-culture system, we pre-treated the system with different OCR concentrations for $24 \mathrm{~h}$ and tested. VEGFa mRNA expression in HUVECs in the co-culture system increased significantly compared with HUVECs cultured alone $(\mathrm{P}<0.001)$. When compared with the co-culture control group (co-culture with no OCR treatment), the OCR $50 \mu \mathrm{g} / \mathrm{mland} 150$ $\mu \mathrm{g} / \mathrm{ml}$ intervention groups significantly reduced VEGFa mRNA expression in the co-culture system $(\mathrm{P}<0.01, \mathrm{P}<0.05)$. These data suggested that co-culturing with HCT116 cells increased VEGFa mRNA expression in HUVECs, and the intervention of 
an OCR dose $(50 \mu \mathrm{g} / \mathrm{ml}$ and/or $150 \mu \mathrm{g} / \mathrm{ml})$ reduced this expression (Figure 4B).

To further investigate the effects of OCR on VEGFa protein expression in HUVECs in the co-culture system, the system was pre-treated with different OCR concentrations for $24 \mathrm{~h}$. Western blotting showed that VEGFa protein expression in co-cultured 50 $\mu \mathrm{g} / \mathrm{ml}$ and $150 \mu \mathrm{g} / \mathrm{ml}$ OCR groups was significantly lower than the co-cultured control group $(\mathrm{P}<0.001)$. In addition, VEGFa protein expression in the $150 \mu \mathrm{g} / \mathrm{ml}$ co-culture group was significantly lower than the $50 \mu \mathrm{g} / \mathrm{ml}$ co-culture group $(\mathrm{P}<0.001)$ (Figures 4C, D). These effects were concentration-dependent.

\section{The Inhibitory Effects of OCR on Subcutaneously Transplanted Colon Cancer Tumours}

We investigated the effects of OCR on the general state and weight of nude mice. After cells were subcutaneously transplanted, mouse weight was recorded every 3 days. The ratio of body weight to initial body weight after transplantation was taken as the weight multiple of the nude mice. Weight multiple results showed that the weight of nude mice in each group increased variably within 15 days of subcutaneous transplantation; the weight of nude mice in each group decreased to different degrees after 15 days. We observed no differences in this trend between groups, which may have been related to tumour growth (Figure 5A).

As specified, tumour growth was recorded every 3 days after transplantation. Tumour volume change data for each group suggested no significant differences between groups within 15 days of transplantation. After OCR administration, tumour growth appeared to differentiate between groups (Figures 5B, C). We observed that tumour volume in OCR and combination groups increased slowly.

\section{The Effects of OCR Blood Vessel Normalisation in Tumours}

When compared with the model group, the number of microangiogenesis vessels which was labelled by $\mathrm{CD} 31$ in the other groups was significantly reduced $(\mathrm{P}<0.001)$. Also, the number of microangiogenesis vessels in tumours in the OCR + oxaliplatin group were significantly lower than OCR and oxaliplatin groups $(\mathrm{P}<0.05)$ (Figures 5D, E). These results suggested that both OCR and oxaliplatin inhibited microangiogenesis vessel formation in tumours, whereas combined use exerted better inhibitory effects in tumour vessels.

Compared with the model group, PDGFR positive rates in tumour vessels in the other groups significantly increased $(\mathrm{P}<$ 0.001). In addition, PDGFR positive rates in the OCR + oxaliplatin group were significantly higher than individual oxaliplatin groups $(\mathrm{P}<0.05)$ (Figures $\mathbf{6 A}, \mathbf{B})$. These results suggested that both OCR and oxaliplatin increased PDGFR coverage in tumour perivascular cells.

Compared with the model group, the positive rates for vascular endothelial VE-cadherin in tumour vessels of the other groups were significantly increased (Figures 6C, D). Also, the positive rate for VE-cadherin in the OCR +
A

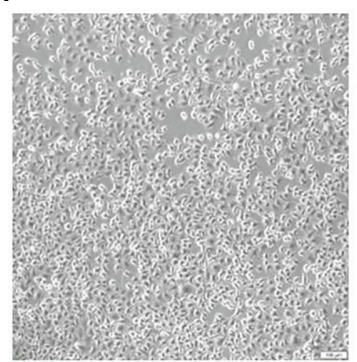

Co-culture Control

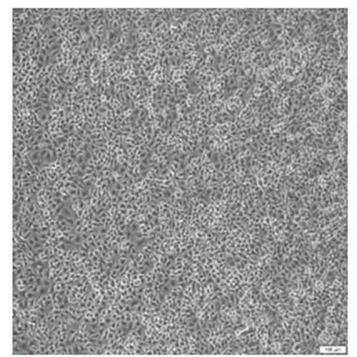

Co-culture 50

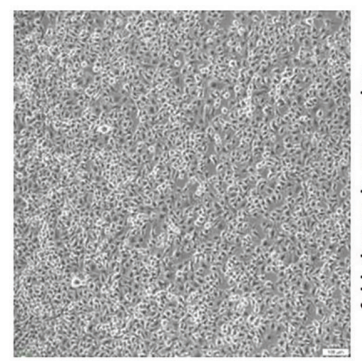

Co-culture 20

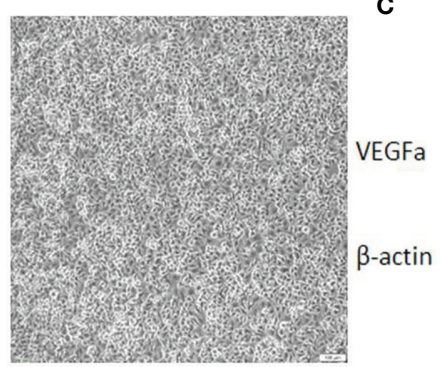

Co-culture 150

\section{B}
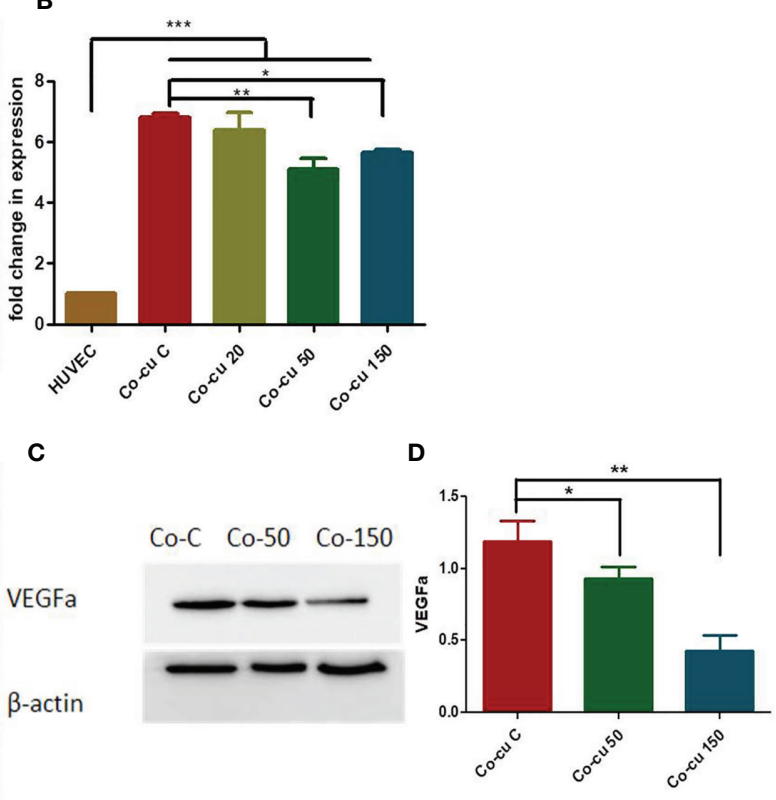

FIGURE 4 | The effects of essential oil from Curcumae rhizoma (CR) (OCR) on HUVECs co-cultured with HCT116 cells. (A) Changes in HUVEC morphology in the co-culture system at different OCR concentrations. (B) The effects of OCR on VEGFa mRNA expression in HUVECs. (C, D) The effects of OCR on VEGFa protein expression in HUVECs. $\left(n=3\right.$, mean \pm standard deviation $(S D),{ }^{* \star} P<<0.001,{ }^{* \star} P<0.01,{ }^{*} P<0.05$ compared with the control group). 
A
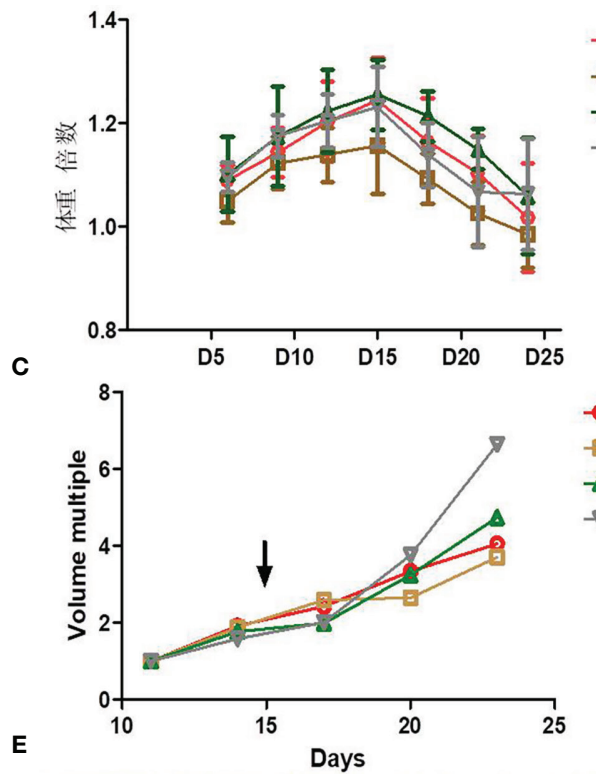

B

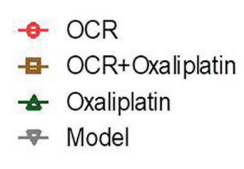

- OCR

- OCR+Oxaliplatin

Oxaliplatin

$\rightarrow$ Control

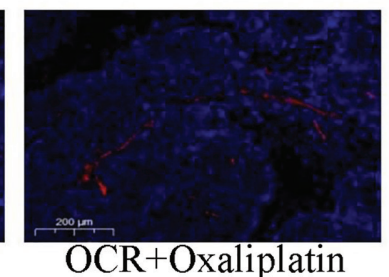

D
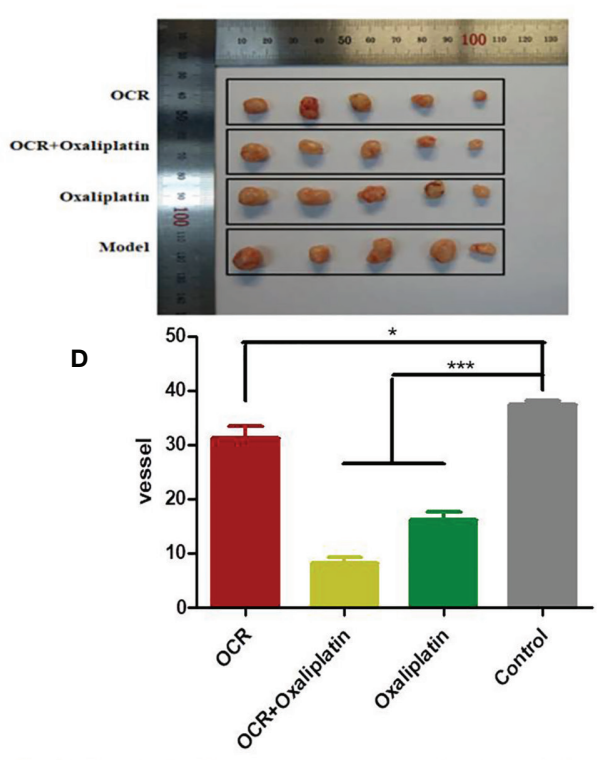

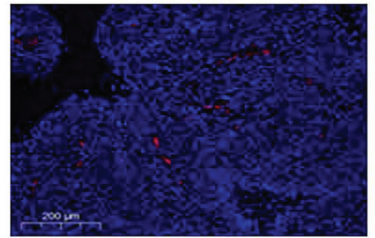

Oxaliplatin

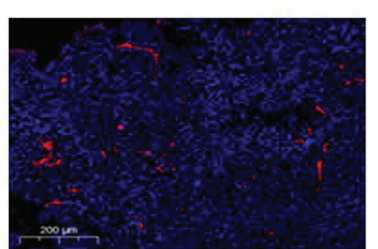

Model

FIGURE 5 | The inhibitory effects of essential oil from Curcumae rhizoma (CR) (OCR) on subcutaneously transplanted colon cancer tumours. (A) Changes in mice body weight in different groups. (B, C) Tumour size in different groups. (D, E) CD31 marker expression in tumours in different groups. ( $\mathrm{n}=5$, mean \pm standard deviation (SD), ${ }^{\star \star \star} \mathrm{P}<0.001$, ${ }^{\star} \mathrm{P}<0.05$ compared with the control group).

oxaliplatin group was significantly higher than the individual OCR and oxaliplatin groups. These results suggested that when combined, OCR and oxaliplatin increased VE-cadherin coverage in tumour vessels.

\section{DISCUSSION}

Previous studies have shown that OCR exerts anti-cancer, antiinflammatory, liver protection, immune regulation and chemoprevention effects (17). However, no systematic research has yet shown the effects of OCR on tumour vessel normalisation. Therefore, we explored whether OCR inhibited tumour growth by regulating tumour vessels.

OCR elicited inhibitory effects on HUVEC and HCT116 proliferation, with the effects in HCT116 cells concentrationdependent. Also, when compared with HCT116 cells, HUVECs were more resistant to OCR. This tolerance difference between normal and disease model cells suggested OCR exerted a unique intervention effect on diseases. Equally, drug dosage is a great concern for clinicians; we identified that OCR dosage differences in inhibiting normal cell activity and disease model cell activity was a distinct OCR advantage. Thus, when selecting reasonable therapeutic dosages, clinicians can theoretically protect normal cells while inhibiting diseased cells.

VEGFa is an important angiogenic factor. Its vascular permeability allows macromolecular proteins such as fibrinogen to leak from blood vessels, thereby forming capillary blood networks (5). Also, VEGFa selectively acts on vascular endothelial cells to promote proliferation and migration, to ultimately form new blood vessels (21). In the tumour environment, massive tumour metabolic consumption, severe local tumour hypoxia and large metabolite accumulation feeds back to stimulate tumour cell secretion of a variety of angiogenesis-related factors, thereby rapidly forming new blood vessels. VEGFa is one such fast-response angiogenic factor (22). Studies have confirmed VEGFa is highly expressed in various tumours in the liver, breast, colon and melanoma cancer (23-27). We observed that OCR inhibited VEGF RNA and protein expression in HUVECs. When co-cultured with colon cancer cells, HUVECs displayed abnormal morphology. Also, VEGFa expression in HUVECs in the disease co-culture model was significantly higher than in HUVECs cultured alone.

These data verified the traditional tumour vessel theory to a certain extent. During HUVEC co-cultivation with HCT116 cells, these cells secreted molecules that acted on HUVECs, thereby 


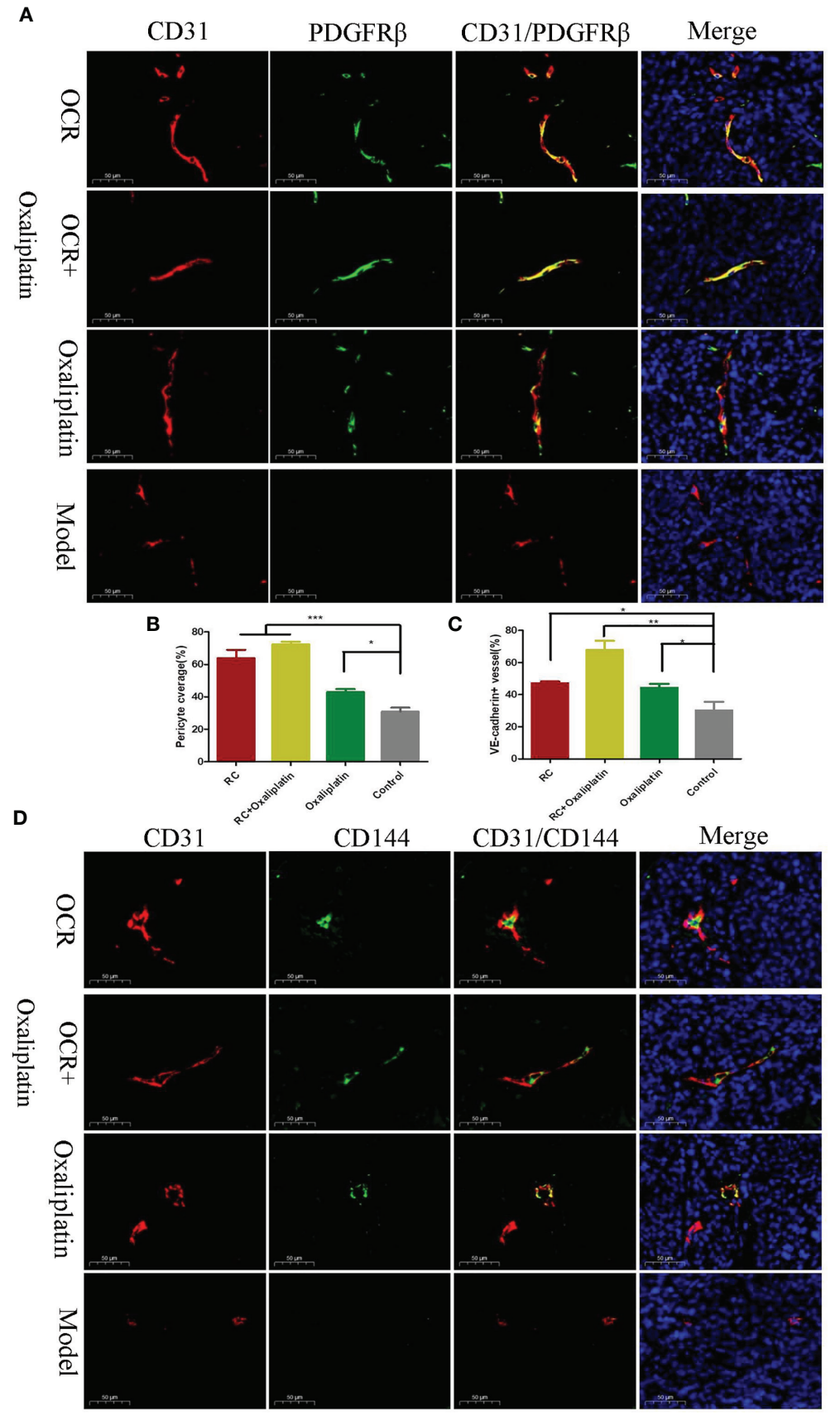

FIGURE 6 | The effects of essential oil from Curcumae rhizoma (CR) (OCR) on tumour blood vessel normalisation. (A, B) Tumour pericyte coverage in different groups. (C, D) VE-cadherin tumour coverage in different groups. $\left(n=5\right.$, mean \pm standard deviation $(S D)$, ${ }^{\star \star \star} P<0.001$, ${ }^{\star \star} P<0.01$, ${ }^{\star} P<0.05$ compared with the control group).

facilitating a significant increase in VEGFa mRNA expression. This observation indicated that the co-culture system accurately reflected changes in endothelial cells during tumour pathology. Our data also suggested that an OCR intervention $(50 \mu \mathrm{g} / \mathrm{ml}$ and $150 \mu \mathrm{g} / \mathrm{ml})$ reversed this rapid increase in VEGFa RNA expression in HUVECs. Western blotting evidence also suggested that OCR $(50 \mu \mathrm{g} / \mathrm{ml}$ and $150 \mu \mathrm{g} / \mathrm{ml}$ ) treatments significantly reduced VEGFa protein expression in HUVECs cultured alone and co-cultured HUVECs. Importantly, these inhibitory effects were concentration-dependent. Therefore, these in vitro investigations suggested that OCR may inhibit endothelial cells' overgrowth during cancer pathological conditions.

Many factors in the body promote angiogenesis, with compensatory mechanisms operating under certain conditions 


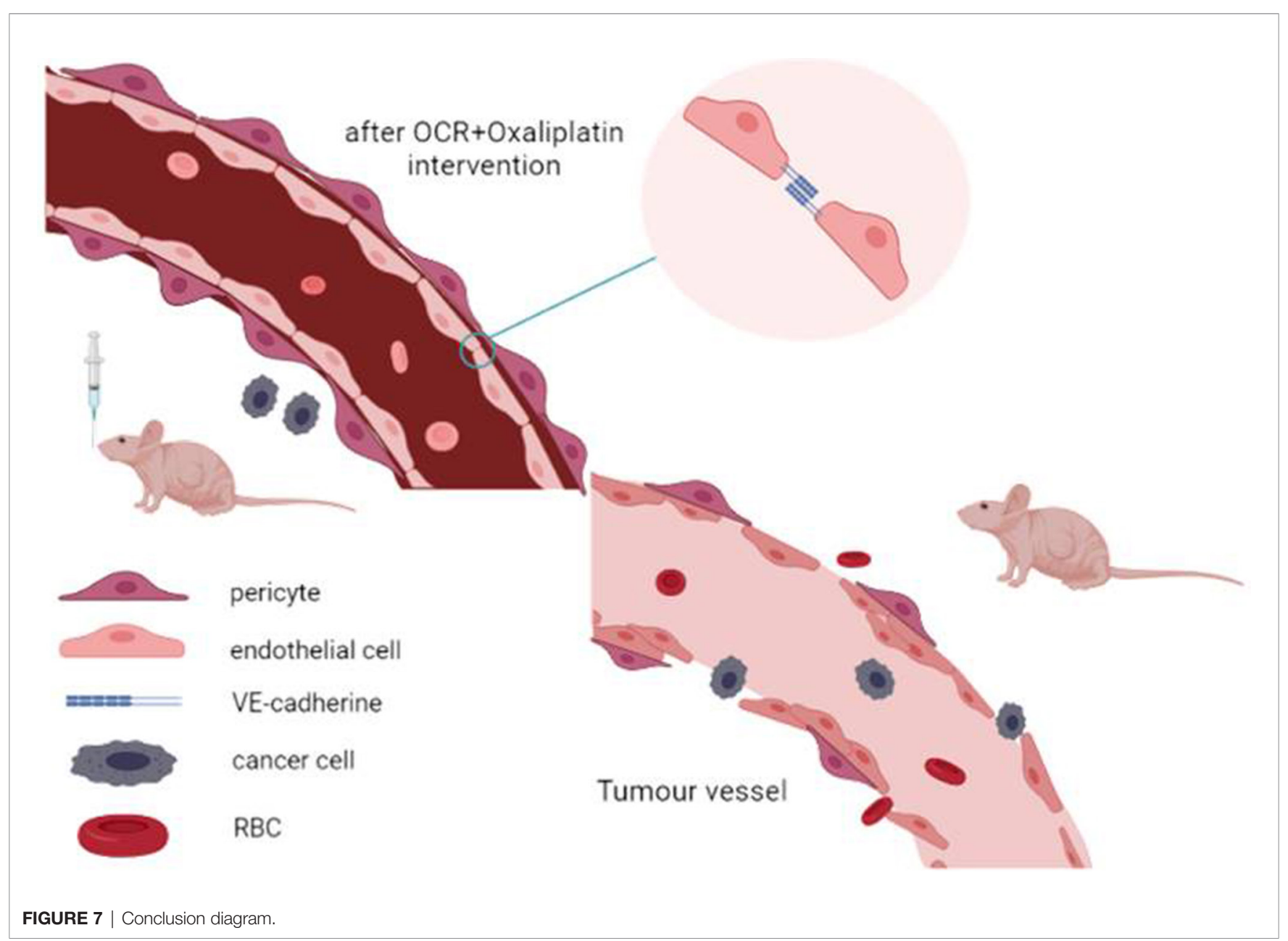

(28). Currently, no single characteristic factor reflects the maturity of tumour vessels (29). Therefore, to further dissect the effects of OCR on tumour vessels and closely observe these effects on tumour vessel morphology and structure, we conducted comprehensive in vivo studies. Our weight results in mice showed that OCR combined with oxaliplatin had no significant effects on these data. Also, tumour volume changes in mice showed that individual OCR and combined with oxaliplatin slowed down tumour growth rates.

According to tumour vessel characteristics, reducing tumour angiogenesis rates and improving vessel structure are effective strategies in normalising tumour vessels. Therefore, we explored the impact of OCR on blood vessels in solid tumours. Platelet endothelial cell adhesion molecule-1 (CD31) is located at tight junctions between vascular endothelial cells and may be used to assess tumour angiogenesis. Studies have shown CD31 is expressed in both mature and immature tumour vessels. When compared with other endothelial cell marker factors, CD31 more accurately reflected the number and density of tumour microvessels (30). Our CD31 data indicated that the number of microangiogenesis vessels in the model group was higher than other groups. This was consistent with the literature where blood vessels are rapidly and disorderly formed in tumours. In our work, OCR reduced the numbers of microangiogenesis vessels in tumours, whereas the combination with oxaliplatin increased them. In addition, this combination altered disordered tumour microvessels to continuous and orderly vessels.

Pericytes are important components of arterioles, veins, capillaries (31), providing support for endothelial cells (32). Within the vascular structure, endothelial cells surround to form a lumen. Pericytes adhere to endothelial cells and the basement membrane which surrounds them (33). Thus, pericytes cover endothelial cells and help quiescent endothelial cells maintain a non-proliferative state. However, during tumour growth, pericytes lose their monitoring capabilities which cause endothelial cells to become activated in the presence of stimulating angiogenic factors (34). Studies have shown that it is vital to restore pericyte coverage in the vasculature in a tumour state (35). From our study, OCR appeared to restore perivascular cell coverage in the tumour, and similarly, OCR and oxaliplatin combined increased this effect.

VE-cadherin is an endothelial cell membrane protein. Physiologically, it regulates contact inhibition between vascular endothelial cells (36). VE-cadherin also generates a dynamic balance between endothelial cell growth and perictyte coverage in blood vessels and helps maintain blood vessel homeostasis. 
Also, VE-cadherin regulates the growth rate of vascular endothelial cells by regulating angiogenic factors $(37,38)$. Studies have shown that VE-cadherin knockout mice experience difficulties forming initial continuous blood vessels due to adhesion barriers between endothelial cells, causing death within 10 days of embryonic development (39). Similarly, in VEcadherin knockout newborn mice, new endothelial cells cannot connect to form new blood vessels, whereas existing endothelial cells in original blood vessels were released (40). Under normal physiological conditions, VE-cadherin is tightly connected to endothelial cells so that cells are ordered and structurally complete. When VE-cadherin expression is lacking (knocked out), endothelial cells cannot undergo monolayer formation and arrangement. In our study, OCR improved VE-cadherin coverage in tumours, and similarly, when combined with oxaliplatin, these effects were enhanced. Thus, OCR inhibited tumour growth, reduced tumour angiogenesis and improved abnormal vascular structures in mouse tumours.

\section{CONCLUSIONS}

OCR inhibited HCT116 and HUVEC activity, proliferation and also VEGFA mRNA and protein expression in HUVECs. It reversed VEGFA mRNA and protein overexpression in HUVEC cells under pathological condition. OCR also inhibited subcutaneously transplanted colon cancer tumour growth rates in mice. Finally, OCR combined with oxaliplatin reduced tumour angiogenesis and increased VE-cadherin and pericyte coverage, thus normalising tumour vascular structure (Figure 7).

\section{REFERENCES}

1. Sajib S, Zahra FT, Lionakis MS, German NA, Mikelis CM. Mechanisms of Angiogenesis in Microbe-Regulated Inflammatory and Neoplastic Conditions. Angiogenesis (2018) 21:1-14. Germany. doi: 10.1007/s10456-017-9583-4

2. Li F, Xu J, Liu S. Cancer Stem Cells and Neovascularization. Cells (2021) 10:1070. Switzerland. doi: 10.3390/cells10051070

3. Movahed ZG, Yarani R, Mohammadi P, Mansouri K. Sustained Oxidative Stress Instigates Differentiation of Cancer Stem Cells Into Tumor Endothelial Cells: Pentose Phosphate Pathway, Reactive Oxygen Species and Autophagy Crosstalk. BioMed Pharmacother (2021) 139:111643. France. doi: 10.1016/ j.biopha.2021.111643

4. Latacz E, Caspani E, Barnhill R, Lugassy C, Verhoef C, Grünhagen D, et al. Pathological Features of Vessel Co-Option Versus Sprouting Angiogenesis. Angiogenesis (2020) 23:43-54. Germany. doi: 10.1007/s10456-019-09690-0

5. Domingues A, Fantin A. Neuropilin 1 Regulation of Vascular Permeability Signaling. Biomolecules (2021) 11:666. Switzerland. doi: 10.3390/biom11050666

6. Folkman J. Tumor Angiogenesis: Therapeutic Implications. $N$ Engl J Med (1971) 285:1182-6. United States. doi: 10.1056/NEJM197111182852108

7. Ramjiawan RR, Griffioen AW, Duda DG. Anti-Angiogenesis for Cancer Revisited: Is There a Role for Combinations With Immunotherapy? Angiogenesis (2017) 20:185-204. doi: 10.1007/s10456-017-9552-y

8. Viallard C, Larrivée B. Tumor Angiogenesis and Vascular Normalization: Alternative Therapeutic Targets. Angiogenesis (2017) 20:409-26. Germany. doi: 10.1007/s10456-017-9562-9

9. Kim SJ, Jung KH, Son MK, Park JH, Yan HH, Fang Z, et al. Tumor Vessel Normalization by the PI3K Inhibitor HS-173 Enhances Drug Delivery. Cancer Lett (2017) 403:339-53. Ireland. doi: 10.1016/j.canlet.2017.06.035

\section{DATA AVAILABILITY STATEMENT}

The raw data supporting the conclusions of this article will be made available by the authors, without undue reservation.

\section{ETHICS STATEMENT}

The animal study was reviewed and approved by Animal Ethics Committee of Chengdu University of Traditional Chinese Medicine (Animal Ethics Approval Number: 2017-08).

\section{AUTHOR CONTRIBUTIONS}

YF: Validation, methodology, and writing-original draft. LD: Methodology. HG: Methodology. YZ: Validation. FP: Conceptualization, writing-reviewing. and editing. GW: Writingreviewing and editing ideas. CY: Conceptualization, writingreviewing and editing, and project administration. All authors contributed to the article and approved the submitted version.

\section{FUNDING}

This work was supported by the National Natural Science Foundation of China (No.81673878), Experimental Formulary Sichuan Youth Science and technology Innovation research team (2020JDTD0022), and Youth Innovation Promotion Association of the Chinese Academy of Sciences (CACM-2020-QNRC1-01).

10. Liu M, Li H, Wang X, Jing L, Jiang P, Li Y. Experimental Study of the Vascular Normalization Window for Tumors Treated With Apatinib and the Efficacy of Sequential Chemotherapy With Apatinib in Lung Cancer-Bearing Mice and Patients. Cancer Med (2020) 9:2660-73. doi: 10.1002/cam4.2923

11. Rabender CS, Bruno N, Alam A, Sundaresan G, Zweit J, Mikkelsen RB. Sepiapterin Enhances Tumor Radio- and Chemosensitivities by Promoting Vascular Normalization. J Pharmacol Exp Ther (2018) 365:536-43. United States. doi: 10.1124/jpet.117.245258

12. Huang Y, Goel S, Duda DG, Fukumura D, Jain RK. Vascular Normalization as an Emerging Strategy to Enhance Cancer Immunotherapy. Cancer Res (2013) 73:2943-8. doi: 10.1158/0008-5472.CAN-12-4354

13. Peng F, Xiong L, Xie X, Tang H, Huang R, Peng C. Isoliquiritigenin Derivative Regulates miR-374a/BAX Axis to Suppress Triple-Negative Breast Cancer Tumorigenesis and Development. Front Pharmacol (2020) 11:378. doi: 10.3389/fphar.2020.00378

14. Peng F, Xiong L, Peng C. (-)-Sativan Inhibits Tumor Development and Regulates miR-200c/PD-L1 in Triple Negative Breast Cancer Cells. Front Pharmacol (2020) 11:251. doi: 10.3389/fphar.2020.00251

15. Peng F, Tang H, Du J, Chen J, Peng C. Isoliquiritigenin Suppresses EMTInduced Metastasis in Triple-Negative Breast Cancer Through miR-200c/CJUN/[Formula: See Text]-Catenin. Am J Chin Med (2021) 49:505-23 Singapore. doi: 10.1142/S0192415X21500233

16. Wang L, Peng F, Peng C, Du J-R. Gut Microbiota in Tumor Microenvironment: A Critical Regulator in Cancer Initiation and Development as Potential Targets for Chinese Medicine. Am J Chin Med (2021) 49:609-26. Singapore. doi: 10.1142/S0192415X21500270

17. Chen Y, Zhu Z, Chen J, Zheng Y, Limsila B, Lu M, et al. Terpenoids From Curcumae Rhizoma: Their Anticancer Effects and Clinical Uses on 
Combination and Versus Drug Therapies. BioMed Pharmacother (2021) 138:111350. France. doi: 10.1016/j.biopha.2021.111350

18. Feng Y, Zhao Y, Li Y, Peng T, Kuang Y, Shi X, et al. Inhibition of Fibroblast Activation in Uterine Leiomyoma by Components of Rhizoma Curcumae and Rhizoma Sparganii. Front Public Heal (2021) 9:650022. doi: 10.3389/ fpubh.2021.650022

19. Lin L, Ge-Er L, Fang-Zhou Y, Bao-Chang C, Chunqin M, Tu-Lin L, et al. Metabolomic Profiling of the Effects of Curcumae Rhizoma and Sparganii Rhizome on Stress-Led Blood Stasis. Pak J Pharm Sci (2018) 31:333-9. Pakistan.

20. Chen P, Li X, Zhang R, Liu S, Xiang Y, Zhang M, et al. Combinative Treatment of $\beta$-Elemene and Cetuximab is Sensitive to KRAS Mutant Colorectal Cancer Cells by Inducing Ferroptosis and Inhibiting Epithelial-Mesenchymal Transformation. Theranostics (2020) 10:5107-19. doi: 10.7150/thno.44705

21. Dragoni S, Turowski P. Polarised VEGFA Signalling at Vascular BloodNeural Barriers. Int J Mol Sci (2018) 19:1378. doi: 10.3390/ijms19051378

22. Claesson-Welsh L, Welsh M. VEGFA and Tumour Angiogenesis. J Intern Med (2013) 273:114-27. England. doi: 10.1111/joim.12019

23. Llovet JM. Focal Gains of VEGFA: Candidate Predictors of Sorafenib Response in Hepatocellular Carcinoma. Cancer Cell (2014) 25:560-2. doi: 10.1016/j.ccr.2014.04.019

24. Sa-Nguanraksa D, O-Charoenrat P. The Role of Vascular Endothelial Growth Factor a Polymorphisms in Breast Cancer. Int J Mol Sci (2012) 13:14845-64. doi: 10.3390/ijms131114845

25. Negri F, Bozzetti C, Pedrazzi G, Azzoni C, Bottarelli L, Squadrilli A, et al. High Levels of Notch Intracellular Cleaved Domain are Associated With Stemness and Reduced Bevacizumab Efficacy in Patients With Advanced Colon Cancer. Oncol Rep (2019) 42:2750-8. Greece. doi: 10.3892/or.2019.7349

26. Bednarz-Misa I, Diakowska D, Szczuka I, Fortuna P, Kubiak A, Rosińczuk J, et al. Interleukins 4 and 13 and Their Receptors Are Differently Expressed in Gastrointestinal Tract Cancers, Depending on the Anatomical Site and Disease Advancement, and Improve Colon Cancer Cell Viability and Motility. Cancers (Basel) (2020) 12:1463. doi: 10.3390/cancers12061463

27. Song Y, Lu H, Wang Q, Xiang R. Targeting Angiogenesis by Blocking the ATMSerRS-VEGFA Pathway for UV-Induced Skin Photodamage and Melanoma Growth. Cancers (Basel) (2019) 11:1847. doi: 10.3390/cancers11121847

28. Viallard C, Audiger C, Popovic N, Akla N, Lanthier K, Legault-Navarrete I, et al. BMP9 Signaling Promotes the Normalization of Tumor Blood Vessels. Oncogene (2020) 39:2996-3014. England. doi: 10.1038/s41388-020-1200-0

29. Goel S, Duda DG, Xu L, Munn LL, Boucher Y, Fukumura D, et al. Normalization of the Vasculature for Treatment of Cancer and Other Diseases. Physiol Rev (2011) 91:1071-121. doi: 10.1152/physrev.00038.2010

30. Sumpio BE, Yun S, Cordova AC, Haga M, Zhang J, Koh Y, et al. MAPKs (ERK1/ 2, P38) and AKT can be Phosphorylated by Shear Stress Independently of Platelet Endothelial Cell Adhesion Molecule-1 (CD31) in Vascular Endothelial Cells. J Biol Chem (2005) 280:11185-91. United States. doi: 10.1074/jbc.M414631200

31. Rajendran S, Seetharaman S, Dharmarajan A, Kuppan K. Microvascular Cells: A Special Focus on Heterogeneity of Pericytes in Diabetes Associated
Complications. Int J Biochem Cell Biol (2021) 134:105971. Netherlands. doi: 10.1016/j.biocel.2021.105971

32. Delprat V, Michiels C. A Bi-Directional Dialog Between Vascular Cells and Monocytes/Macrophages Regulates Tumor Progression. Cancer Metastasis Rev (2021). Netherlands. doi: 10.1007/s10555-021-09958-2

33. Caporarello N, D’Angeli F, Cambria MT, Candido S, Giallongo C, Salmeri M, et al. Pericytes in Microvessels: From "Mural" Function to Brain and Retina Regeneration. Int J Mol Sci (2019) 20:6351. doi: 10.3390/ijms20246351

34. Burdyga T, Borysova L. Ca(2+) Signalling in Pericytes. Adv Exp Med Biol (2018) 1109:95-109. United States. doi: 10.1007/978-3-030-02601-1_8

35. Park JS, Kim IK, Han S, Park I, Kim C, Bae J, et al. Normalization of Tumor Vessels by Tie2 Activation and Ang2 Inhibition Enhances Drug Delivery and Produces a Favorable Tumor Microenvironment. Cancer Cell (2016) 30:95367. doi: 10.1016/j.ccell.2016.10.018 Elsevier Inc.

36. Lagendijk AK, Hogan BM. VE-Cadherin in Vascular Development: A Coordinator of Cell Signaling and Tissue Morphogenesis. Curr Top Dev Biol (2015) 112:325-52. United States. doi: 10.1016/bs.ctdb.2014.11.024

37. Seebach J, Klusmeier N, Schnittler H. Autoregulatory "Multitasking" at Endothelial Cell Junctions by Junction-Associated Intermittent Lamellipodia Controls Barrier Properties. Front Physiol (2020) 11:586921. doi: 10.3389/ fphys.2020.586921

38. Giannotta M, Trani M, Dejana E. VE-Cadherin and Endothelial Adherens Junctions: Active Guardians of Vascular Integrity. Dev Cell (2013) 26:441-54. United States. doi: 10.1016/j.devcel.2013.08.020

39. Carmeliet P, Collen D. Molecular Basis of Angiogenesis. Role of VEGF and VE-Cadherin. Ann N Y Acad Sci (2000) 902:244-9. United States. doi: 10.1111/j.1749-6632.2000.tb06320.x

40. Murakami M, Nguyen LT, Zhuang ZW, Moodie KL, Carmeliet P, Stan RV, et al. The FGF System has a Key Role in Regulating Vascular Integrity. J Clin Invest (2008) 118:3355-66. doi: 10.1172/JCI35298

Conflict of Interest: The authors declare that the research was conducted in the absence of any commercial or financial relationships that could be construed as a potential conflict of interest.

Publisher's Note: All claims expressed in this article are solely those of the authors and do not necessarily represent those of their affiliated organizations, or those of the publisher, the editors and the reviewers. Any product that may be evaluated in this article, or claim that may be made by its manufacturer, is not guaranteed or endorsed by the publisher.

Copyright $\odot 2021$ Feng, Deng, Guo, Zhao, Peng, Wang and Yu. This is an open-access article distributed under the terms of the Creative Commons Attribution License (CC BY). The use, distribution or reproduction in other forums is permitted, provided the original author(s) and the copyright owner(s) are credited and that the original publication in this journal is cited, in accordance with accepted academic practice. No use, distribution or reproduction is permitted which does not comply with these terms. 